\title{
Anesthetic management using extracorporeal circulation support of a patient with a great lung mass complicated by cardiac tamponade
}

Freire Otero M., Álvarez Zancada E., Santiago Paniagua P., Cuéllar Bobadilla C., Blanco Pieschacón D., Álvarez-Rementería Carbonell R.

Fundación Jiménez Díaz University Hospital, Dept of Anaesthesiology \& Pain Medicine, Madrid, Spain

Background: Extracorporeal circulation (ECC) is primarily used for open heart surgery, however, there are also other medical and surgical conditions in which could be useful. ECC is occasionally used to ensure adequate oxygenation and hemodynamic stability during thoracic, brain, liver or kidney surgeries ${ }^{1}$.

Case report: A 68 year old male patient was admitted to the Emergency Department of our institution with dyspnea and cough associated with chest pain and hemodynamic instability. Chest X-ray and CT shows mediastinal shift toward the left side due to a large mass in right hemithorax associated with pleural and pericardial effusion. Surgery was scheduled for removal of the mass. When the patient arrived at the operative theater left radial artery and right internal jugular vein were cannulated, subsequently, and after administration of heparin $(300 \mathrm{IU} / \mathrm{kg})$ and measurement of the activated clotting time, femoral vessels were cannulated under local anesthesia. The venous cannula was placed in the right atrium and the arterial cannula in the iliac artery. Then extracorporeal circulation was initiated, anesthesia was induced and the patient intubated. Thoracotomy was performed finding a tumor of $40 \mathrm{~cm} \times 30 \mathrm{~cm}$, with important neovascularization. The tumor was fixed to the mediastinum, pericardium, pleura and diaphragm, also presenting pleural and pericardial effusion. Complete resection of the tumor was performed.
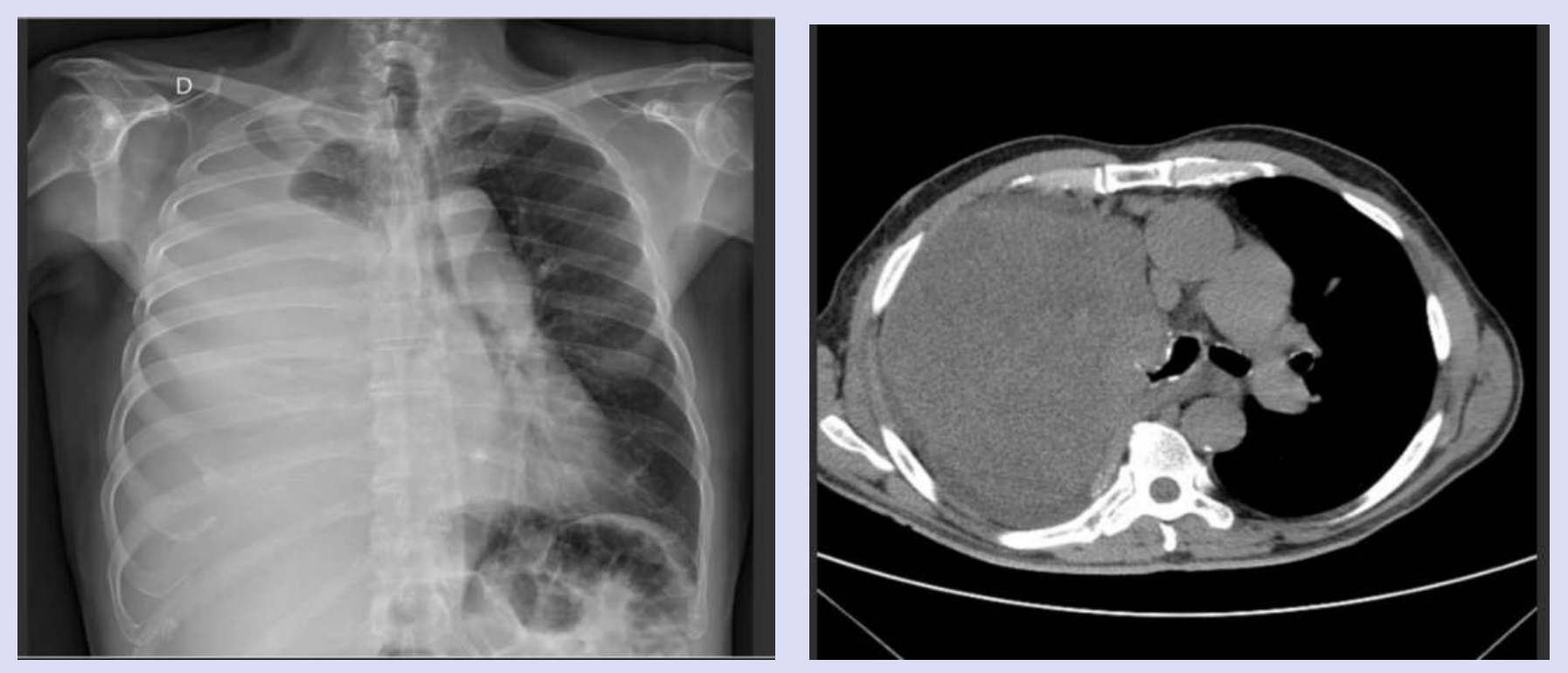

The patient had a torpid postoperative course and he finally deceased due to his injuries.
Discussion: In the current literature there are some reported cases of establishment of ECC before anesthetic induction. We chose to initiate extracorporeal circulation in awake patient to minimize the risk of complications associated with induction of anesthesia and mechanical ventilation in a patient hemodynamically compromised by tumor compression of the right cavities and pericardial effusion.
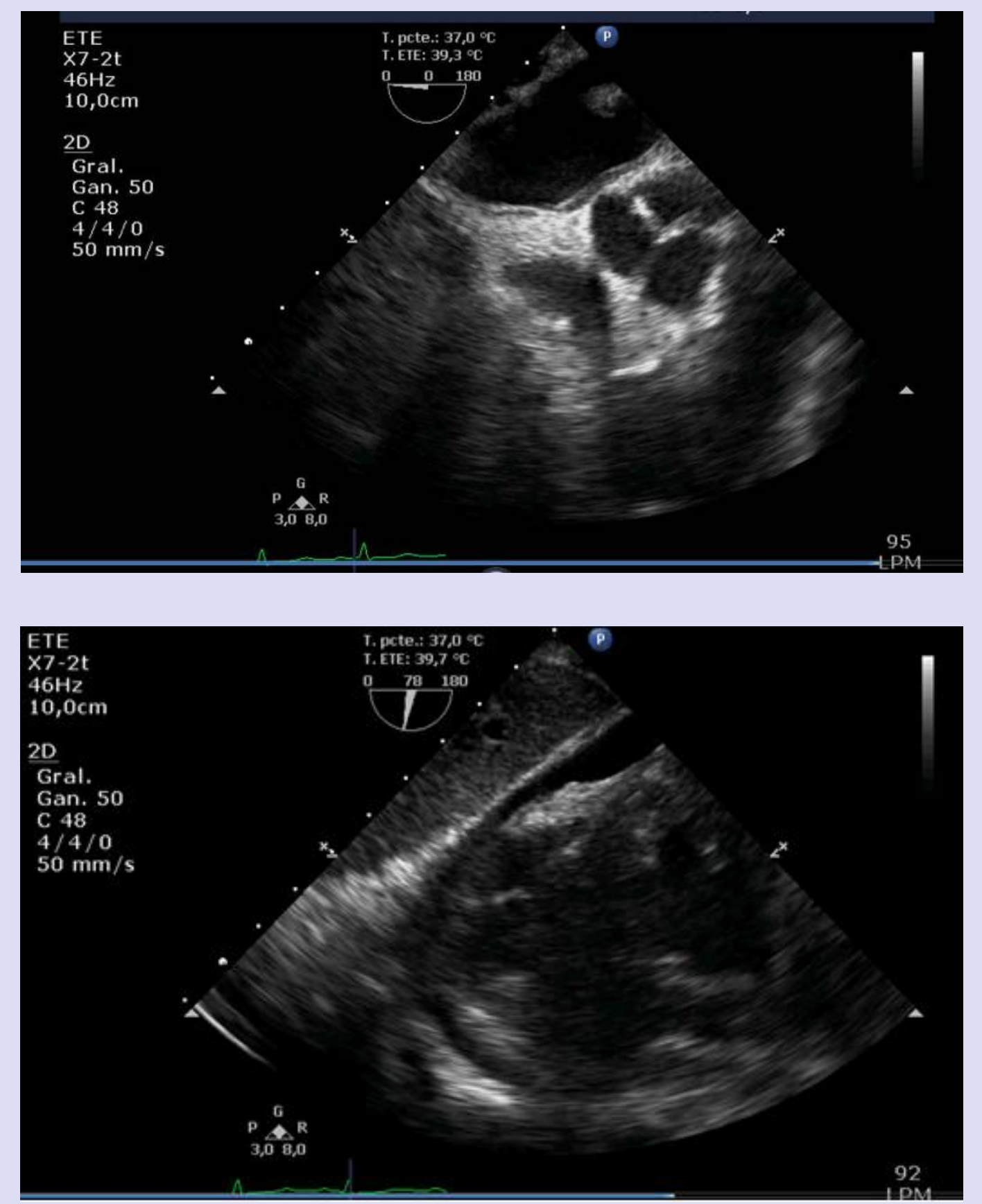

Learning points: With the use of ECC, difficult and complex surgeries can be performed more safely and some seriously ill patients may benefit from establishing extracorporeal circulation under local anesthesia prior to anesthetic induction. It is important that the surgeon, anesthesiologist, and perfusionist are trained in applications of ECC. 